\title{
INDIRIZZO INTRODUTTIVO
}

\author{
alla sessione \\ sulle Ricerche di Meteorologia e Fisica dell'Atmosfera \\ del Presidente di sessione, Generale B. A. Fernando Giansanti, \\ Capo del Servizio Meteorologico dell'A. M. I.
}

(Romn - E. L. R., 13 Gingno 1961)

Ficcellenze, Signore, Signori,

Lat sessione di stamane del Convegno Internazionale Teenico-Seientilico dello Spazio è dedicata alle Ricterehe di Meteorologia. Poiché is toecato a me, quale Cajo del Servizio Meteorologico dell'Aeronautich, l'onore di presiciere questa sessione, mi è grato porgere anzitutto angli scienziati, ai professionisti, agli esperti ed agli appassionati qui convenuti da vari centri di ricerca-e lavoro un eordiale benvenuto ed un rakdo saluto angurale.

Dagli antichissimi parapegmi ad oggi la Meteorologia lia compinto, secolo per secolo, un ben lungo cammino. Tutti ne conosciamo, almeno sommsiannentes, le tappe principali, dal famoso trattato di Aristotele all'invenzione del barometro e del termometro che hamno segnato l'ingresso dei principi della fisiea nello studio dell'atmosfera; son venute poi le prime reti di stazioni, le prime earte sinottiche, il timido esordio dell'esplorazione degli strati aerei a mezzo di paIloni sondat ed aroplant, via via fino alle moderne teorie, alle nuove seoperte, alla previsione numerica del tempo, ai razzi meteorologici ed ai satelliti artificiali de+la famiglia Tiros.

Per quanto lungo e fecondo, questo cammino è ancora ben lungi dall'essere conchiso. Aneora molto resta da fare, prima di poter conoscere a fondo le leggi che regolano l'oceano d'aria the ci avvolge ed i processi che vi si compiono, e prima di poter utilizzare questa ronoscenzit, con tranquilla sieurezza, ai nostri principali fini pratici: tra i quali primeggiano la previsione del tempo - specie quella a lnuga scadenz - e forse, domani, il controllo stesso del tempo da parte dell'uomo.

Non mi è praticamente possibile, come sarebbe invero desiderabile, fare in poohi minuti il punto della situazione delle ricerehe, poiché tanti 
sono i settori e molto vari gli aspetti delle questioni sulle quali la Meteorologia Scientified opera. Non voglio neppure, t'altronde, presumere lli adlitare percorsi e mete agli illustri scienziati qui convenuti.

T'uttavia, la mia fumzione di Capo di un Servizio che la problemi pratici ed applinativi di Breteorologia tanto nrgenti ed importanti, quale la diretia custodia della vita per ma omma imponente pabte dell'uma-

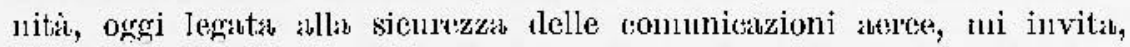
mzi direi quasi ni obbliga, a ricliamare qui aleune questioni che ni apjadiono di fondamentale e genemale interesse. Mi riferisco più particolammente sia ai problemi della Meteorologia informativa e previsionistica, the a queII, appera nascenti, della ALeteorologia sperimentale di intervento.

Tuiziando proprio da questi ultimi ì superfluo ch'io sottolinei come essi siano connessi alla conoscenza apjorofondita della macrofisica e della microfisica delle nubi e delle idrometeore in genere, comprendentovi I'estremamente complessso umpo dei fenomeni eletirici temporalesthi o della clinuica dell’atrnosfera: settori della Bleteorologia che i ricereatiori a gli esperti del mio Servizio vanuo sviluppando con soddisfacente apporto.

La dissoluzione di nebbic, la eccilazione di preoipitazioni, lo sgretoIamento della grandine, ohe ancor oggi si tentano con risultati talvoltia incerti o nulli e spesso molto modesti, potranno, attraverso la ricerci, trovare quelle giuste basi tcoriche dle suggeriranno lo tecniche per operazioni vaste e tempestive, la eni rfficacia, una volta divenuta offettiva, avrà enormi ripercussioni economiche et sociali.

D'altia parte questas comoscenza approfonditab delle idrometeore, della loro genesi e del loro sviluppo, dabla fase delle nubi a quella delle precizitazioni, avrà anche, cone risultato, di facilitare la previsione di questi fenomeni e quindi anclue lab arronavigazione in loro presenzit. L'a]porto informativo diretto ed indiretto ottenibile con radar meteorologiri sulla struttura, dimensioni, distribuzione degli ammassi nuvolosi sarà, sotto questo aspetto, veranente essenziale.

Venetulo ad aspetti che protremmo considerare più olassici detlat Heterologia, specie per quanto righuarda in particolare il nostro Meeliterranco, molto vi è da lavoraure nel oarn] o troposferico e substratosferico. Data lab situazione geografica, in lapporto alla influenza non sempre determinabile delle circolazioni legate alle grandi cellule anticidonichse subtropicali, avviene che il Maditerraneo sia serle di trasformazioni o? al contrasti alelle masse l'aria she lo interessano con le loro traiettorie, ab volte percorrendolo velocemente, a volte sostandovi jer periodi più o meno lurıghi, spesso dando luogo a matteristici processi di genera- 
zione e rigenerazione rlelle perturbazioni che non sempre riusciłmo it chibrile e prevedere con soddisfacente precisione.

Ido studio sbprofonclito di questi problemi di Meteorologia Sinotticta ohe assmmono uspetti mecanici, termodinamici e statistico-elimmtologici delle vicissitudini utmosferiche è stato, avendo present d'enormità degli stessi, rai miei rimerotori appena abbozzato e tentato in asi sporadici; esso però, ovviamente, merita e richicsle il massimo sforzo.

son josso non concordaue, a questo proposito, con l'insistente appello col quale la Organizzazione Meteorologiou Honcliale sollecita um incremento delle osservazioni in quota sulle zone alle basse latitudini. là ove ha sede il maggior apporto di energia por la dinamica a termodinamick della nostra sutmosfera o dove peraltro questa preziosa doculmentazione di informazione o di studio è anrora terribilmente soursib, lassiando troppo eampo a estrajolazioni incerte e spesso anche pericolose.

Je precerlenti ricers:le, poi, si rieollegano strettamente a quelle: sullat rarliazione solare, sulla evaporazione, sulla turbolenza e lo scambio sia nei bassi strati she in quelli superiori; ricerele che risultano essenziali ancle sotto altri punti di vista che vanno dalla radio e redarpropagazione alla dispersione deyli effluenti nuslesuri, e che presentuno problemi di grande dificoltà teorisa, sperimentale exl organizzativa, ma clu: certamente tempo, mezi e volontì varrunno as smperare.

I'incremento notevolissimo dei sondaggi termodinamiei dell'atmosfera, realizato durante la guerra e subito rlopo. lau apportato al progresso delle conoscenze meteorologiche un contributo urandissimo, a prer sottolinesurlo mi basti qui citare le correnti a getto efl il contributo che la. loro utilizzazione puó dare ulla previsionistios.

E pertanto da attendersi che nuovi ed altrettanto importanti contributi vengano da un ulteriore decisivo e sistematico inremento dello attuali quote raggiunte dai sondaggi stessi, aceompagnato ovvianuente dis un arleguamento relle strumentazioni ri misura.

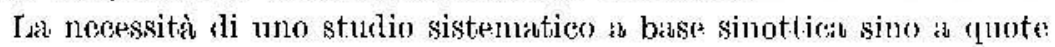
the non si arrestino più ai $15-20 \mathrm{~km}$, ma si sjingano sino ai $30-35 \mathrm{~km}$, oggi raggiungibili con una buona orgmnizzazione, is ress aviflente sulche in sede praticu, se si consider che fra cuattro o einque auni al massimo entreranno in servizio di linea aeroplani progettati per rolare arl oltro 20.000 metri di altezza, con velocità almeno ropplia di auklla del smono.

Tale studio, che interessa ormai l'alta stratosiera ed i primi strati della mesosfera, risulta essenziale ancle per lo viportussioni ohe questa dinamica degli strati superiori deve avere su quelli inferiori, dove si svolge l'attuale volo e nei quali noi viviamo. 
Benohé sia fable prevedere l'insorgere di movi e sempre più comsplessi problemi, d rla sperare altresi che almeno alcuni rlegii attuali trovino una soluzione.

Volgenrlo così la mente a quanto ei è già stato dato di appremrlere sugli strati più elevati rlell'atmosfera, dioé della mesosfera e della termosfera, che sino a ieri sono stati explowati solo con mezzi indiretti erl oggi sono raggiunti son razzi meteorologiei, con sondo spaziali, con satelliti, dobbiamo conclurlere che una esplorazione sistematica di tijo sinotitien ci aprirì nuovi orizzonti.

I complessi fenomeni fotonhimici e fotoelettrici, il aratteristiro comportamento dell'ozono, i fenomeni legati alla rarliazione rosmica, ai raggi $X$ e ad ogni altra specie di radiazione, mettono in moto e sono nel tempo stesso condizionati da grandiosi seambi e trasformazioni energetiche, che morlificano sia la struttiua dellatmosfera sujeriore sia i suoi movimenti. La maggior conoscenza di tutto ciò permetiterà di inquadrave? in modelli unitari e coerenti quei fatti che già sono stati a thatti e solo parzialmente rivelati, and esempio, dalla deriva e dalle distorsioni della scie dolle stelle cadenti, a che si tende a sturliare nei piò minuti rlettagli, some las fatio qui in Italis il nostro Booglio, mediante le nubi al sorlis.

L'importanza, clue rlefinirei integrale, di questo grenere di ricerelo? sull'atmosfera superioro iे confermata dalle iniziative prese dalla Organizzazione Metoorologria Mondiale, che ha già prospettatio a stimola le: joroposte di sturlio sistematico sinottion rlella mesosfora merliante mzzi metrorologiei apjorontati per questo sjecifico seopo.

F sono certo che anche il nostro Paese, ed in particolare l'Aeronantioa, non mancheranno all'appello: posso infatti comunicare che l'organizzazione rli questi land là già avuto inizio.

Chinderó questa mia rapirlissima rassegna ricordanelo quelli ahu potremmo rlefinier i più elevati osservatori meteorologici, messi a disposizione degli sturliosi di fisioa rlell'atmosfrem dei tesniri aneriani: i Tiros, „3i cyuali ho accennato all'inizio del mio dire.

Questi satelliti sono notoriamente posti in orbita a $600-800 \mathrm{~km}$ non taulo per esplorare l'altab atmosfera ivi esistente, quanto per misurare le rudiazioni diffuse orl emesse rlallas superficie terrestre a dablintmosfera, e, soprattutto, per tousmetitere dallalto l'aspetto rlelle formazioni o degli mumissi nuvolosi.

Essi pormettono rli ottenere percio un quadro relativo alla quantità, tipo e distribuzione delle nubi dalle zone via via sorvolate.

Le misura le osservazioni racuolt tramite questi satelliti nei loro suecessivi passagui, poste in relazione allo simultane osservazioni e ai rila- 
vamenti normali e strandinari compiuti al suolo e negli strati atmosferiei sino a $25 \mathrm{~km}$, permetteranno di nvere un qualro d'insieme, sino ad oggri mai ottenuto, dei fenomeni meteorolotrici a grande scalaz nella loro evo. luzione e del bilancio temoualiativo atmosferico azd assi learato.

Ciò comsentirì getto di grinugere ad mus maggion compremsione dei fenomeni stessi a di conseguenza, in partiolare, di mitrloralue la pro. yisione.

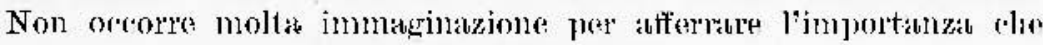
avrebbe, anche dal solo punto di vista pertico informativo, il fatto, la

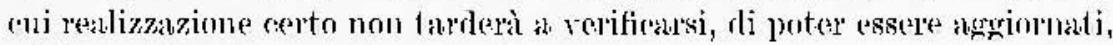
and intervalli di tempo di poro pì di unvora, da un oporortuno eomplesso rli tali satelliti che, con labettorie da polari ad equatoriali, esplorassoro lo stato del rielo su tulta la superficie terrestre.

Ta collaborazione al piano amerirawo di ricerehe è troppo importante

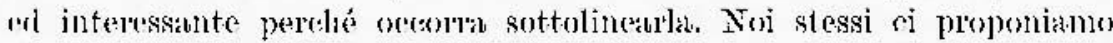
rli "ontribuire nei limiti del possibile.

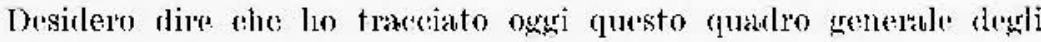
attuali problemi della Meterologria e della Fisica dell'atmosfera, soflermandomi su aleuni problemi della zona del Meditermuen, ron un senso particolare di entusiasmo perohé proprio in questi giomi lo stesso Con-

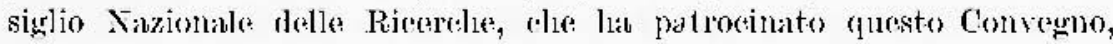
sta coneretando la istituzione di un Centro Nazionale per la Heteorologia a la Fisio dell'atmosfera, Centro ehe si assmumerà il ampito di trattare appunto culesti problemi.

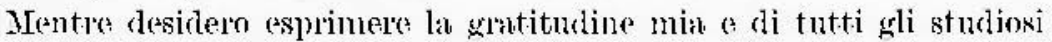
di Heteorologia italiani verso il Presidente del Comsiglio Sizionale dalle Ris'selese, Prof. Polvani, per questa sua gramele miziativa, fuccio roti d'angurio per l'istituendo Contro al quale il Servizio Reteorologico del-

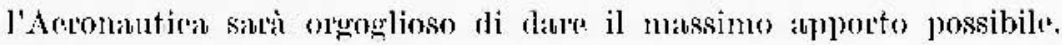

IIo motivi por ritenero de questa informazione salà areolta ron compiacimento anehe dagli studiosi stranieri qui prosenti.

Ma ormai vi ho trattenuto troppo a lungo su considerazioni di alrattele generale; ritorniamo perciò alla nostlä sessiome a diamo inizio allat letturat delle memorite.

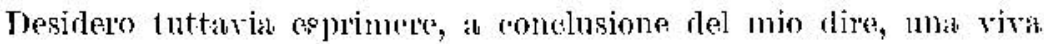
speranza: ahe rai nostri lavori scuturisa non tanto la segnalazione di nuovi problemi da visolvere. quanto la soluzione di qualenono di quelli ai glusli lo fugaremontes aecemmato. 